\title{
21
}

\section{Web Graduate Courses on Benign Urban Water Systems: Optimal Complexity and User Performance Improvement}

\author{
William James and Eduardo Queija de Siqueira
}

Nineteen graduate students from various countries completed in April 2000 a dual graduate course delivered on one web home page. Five professors from four Universities collaborated on this project. The courses (i) urban water pollution control planning and (ii) urban storm water management involved learning certain models, and formed a special asynchronous Internet learning environment. Our experience shows that achievable modeling accuracy depends more on user quality than it does on model structure, and an important concern here is that, although it was not measured in this study, user performance needs to be evaluated objectively and then improved.

\subsection{Introduction}

This chapter covers (i) the idea of writing group decision support systems (GDSS) for models of benign urban water systems in such a way that model users "perform better" than they might otherwise do, and (ii) testing the idea in web-based graduate courses. Ideas for the modeling approach reported here were gleaned from experience gained in previous web graduate courses. We

James, W. and E. Queija de Siqueira. 2002. "Web Graduate Courses on Benign Urban Water Systems: Optimal Complexity and User Performance Improvement." Journal of Water Management Modeling R208-21. doi: 10.14796/JWMM.R208-21.

(C) CHI 2002 www.chijournal.org ISSN: 2292-6062 (Formerly in Best modeling practices for Urban Water Systems. ISBN: 0-9683681-6-6) 
felt that conventional instructional methods do not teach engineers certain skills that are best taught if one draws on the experience of faculty in other countries, and that a new teaching approach was needed.

Among the pedagogic innovations implemented, were attributes that were supposed to improve model user performance. We feel that the question of optimal model complexity is central, and discuss model complexity in some detail.

\subsection{The Internet Learning Environment}

The courses Urban water pollution control planning and Urban storm water management were taught in winter Jan-May 2000, and formed a special asynchronous Internet learning environment (ILE). Learning objectives included to become facile at models that analyze and plan urban water systems, in particular, those that estimate flow and pollutant quantities and optimize conveyance, storage and pollutant removal structures required for storm, sanitary and combined sewer collection systems and the management of the impacts of such systems. Each of the two courses required six modules (one every two weeks), and each module comprised the work set out in Table 21.1. Tables 21.2 and 21.3 set out the content of the various modules in the two courses. Table 21.4 lists the left hand toolbar, developed for all web pages in the course home page system, which was extensive. Fortunately the toolbar neatly encapsulates the main content of the two graduate courses as posted on the web.

Table 21.1 Work required for each module.

\begin{tabular}{lc}
\hline & $6 \mathrm{~h}$ \\
one lecture & $20 \mathrm{~h}$ \\
additional individual work & $10 \mathrm{~h}$ \\
additional technical reading & $8 \mathrm{~h}$ \\
one assignment & $2 \mathrm{~h}$ \\
evaluation of all other student assignments
\end{tabular}

Asynchronous ILE's may be designed to remove the distinctions between instruction, learning and design (work). Readers may review the ILE on the web (the URL must be entered fully in your browser as one line):

/—/WJ661HOMEPAGE.HTML where"/—/" denotes "HTTP://WWW.EOS.

UOGUELPH.CA/WEBFILES/JAMES/HOMEPAGE/TEACHING/661/" 
Table 21.2 Curriculum for 05-661 Urban storm water management modeling.

1. continuous storm water management models and model structure (SWMM and PCSWMM);

3. GIS data management, complexity, discretization and process disaggregation (PCSWMMGIS);

5. routing in complex, looped, partially surcharged pipe/channel networks (SWMM-EXTRAN);

7. pollutant build-up, washoff and transport (SWMM-RUNOFF, TRANS);

9. pollutant removal in sewer networks, storage facilities and treatment plants (DETPOND);

11. Sewer network designs for the future; technologies for wastewater in urban infrastructure.

Table 21.3 curriculum for 05-662 Water pollution control planning

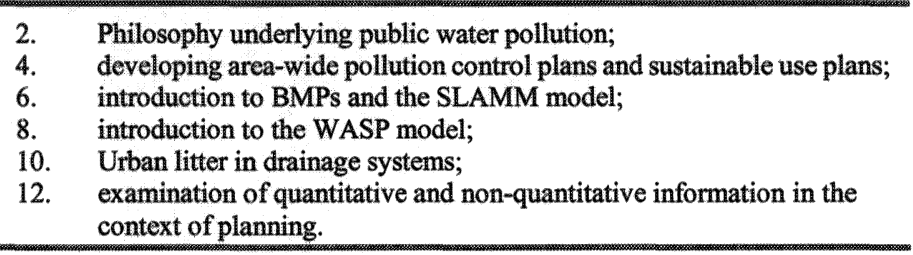

Table 21.4 Left hand toolbar

\begin{tabular}{l}
\hline Daily News \\
* Today/home \\
* Modules \\
* Dates \\
* Class work \\
* Grades \\
* Course ratings \\
\hline Help \\
* Equations \\
* Sample answers \\
* Units \\
* List server \\
* Constants \\
* Abbreviations \\
* Vocabulary \\
* Acronyms \\
* Models \\
* Applets \\
\hline About.... \\
* This site \\
* Course outline \\
* Course index \\
* Questions? \\
\hline Who are we? \\
* Instructors \\
* Thanks \\
* Class list \\
\hline
\end{tabular}

\section{Organization of the courses}

Both courses ran from January till the end of April 2000. The 26 participants came from 11 countries and Provinces (Canada - British Columbia and Ontario, USA, S. Africa, Germany, Brazil, Sweden, Japan, Pakistan, China, France), and four Universities (Guelph, Alabama at Birmingham, Darmstadt and Cape Town). Students elected one or two courses, and their work was posted on the web. The instructors shared instructional activities. Like our first course (James and Ostrowski, 1998), all evaluations were based on web pages developed and evaluated by the participants - students necessarily became facile on the web, Internet, and HTML. Work for each module was spread over two weeks, and all dates were strictly enforced. Five instructors (Bob Pitt, Neil Armitage, Isobel Heathcote, Manfred Ostrowski and Bill James) awarded graduate credit to their own students. The instructor's gradings were also posted on the web. 


\subsection{User Performance Improvement}

Students were required to download documentation and run the executables for WASP, DETPOND, SLAMM and PCSWMMGIS2000. Module M3, the PCSWMMGIS module, is germane; its contents are given in Table 21.5. Model complexity is covered in assignment A3, which is presented in Appendix A.

Table 21.5 Content of module M3.

Introduction
Engineering design and the development of deterministic models
Newtonian mechanics, pde's, fde's and computer programs
Spatial discretization
Temporal resolution
Averaging high-frequency processes
Model complexity
Data collection
GIS
Some urban water systems GIS concepts and terminology
Frequently asked questions
Assignment A3

One innovation included in the dual web graduate courses was the large measure of self-evaluation by the graduate students themselves. Self-evaluation for of each of the assignments and peer evaluations in the two courses was as set out in Tables 21.6 and 21.7. Symbols, assigned the meanings given in Table 21.6, were returned in the self-evaluation exercises carried out by the students for the five questions listed in Table 21.7.

Table 21.6 Self-grading symbols.

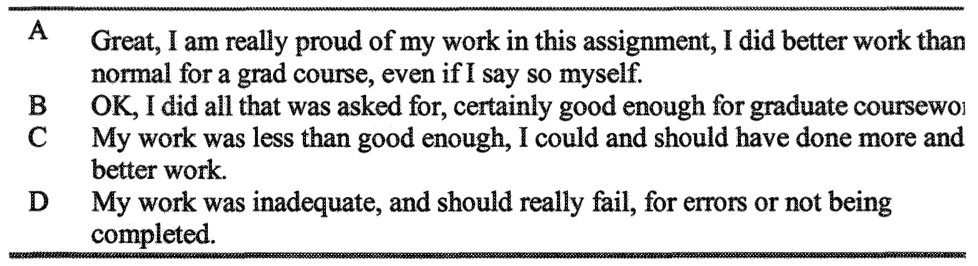

All the above information was collated together with the instructor's gradings. Evidently the students experienced difficulties learning the models, decreasing in the order listed above (WASP most difficult, PCSWMM least difficult). Reasons for their difficulties are clearly complex, but from the detailed students comments, and without offering any convincing factual arguments, it seems evident that the models ranked in increasing learning difficulty for the following reasons: 
Table 21.7 Student self-evaluation exercises.

1 For every one of your own 6 assignments and 6 assignment evaluations in each course, complete the following information:

(a) your self grade (12 symbols required per course)

(b) the number of days late if any that your work was posted (12 integers required per course)

(c) for each of your evaluations only, the number of your peers that you covered (6 integers required per course)

2 Name the one assignment that you did that you consider to have been your best (1 assignment number required per course)

3 Name the assignment evaluation that you did that you consider to have been your best ( 1 evaluation number required per course)

4 Name the peer whose assignment you consider to be the best for each assignment (6 names and assignment numbers required per course)

5 Name the peer (not instructor) whose evaluation of each of your own assignments you consider to have been fairest ( 6 names and evaluation numbers required per course).

1. PCSWMMGIS2000 includes more utilities aimed at user performance improvement,

2. SLAMM has very good user documentation,

3. DETPOND is accompanied by good supporting documents, and

4. WASP is difficult to find, download, install, build data files and run, due to the lack of user help. An older version of the manual was considered more appropriate.

No further objective evaluations of user performance were attempted, other than routine evaluations of assignments.

\subsection{Optimal Model Complexity}

Engineering models of urban water systems are used for planning, design, operation and forensics, listed in increasing complexity. Thousands of different models are being used. Their processes may be classified as physical, chemical, biological and sociological, also in increasing complexity. For an introduction to complexity see for example Flood and Carson (1993), Nihoul (1998) and Beven (1993). User performance and model complexity are inherently and intimately inter-related. Yet few guidelines are available for selecting the best level of complexity for a given application, and similarly few models are programmed to systematically improve user performance. With web-based models these issues need attention, due to wider usage.

Increased understanding of the processes incorporated into model algorithms has over the years led to an increasingly descriptive level of detail and complexity. Several researchers point out that the engineering profession during the last century has been preoccupied with development of more detailed 
deterministic descriptions. Many writers state that it is important to know the appropriate level of complexity required to describe a given system with the minimum level of uncertainty.

Ambrose et al. (1991) provide a table of complexity. At the lowest level, just one source of oxygen (re-aeration) and one sink (biochemical oxygen demand) are considered in the dissolved oxygen budget. Higher levels of complexity include other factors affecting dissolved oxygen concentrations such as nitrogenous biochemical oxygen demand, nitrification and eutrophication. Brooks and Tobias (1996) believe that overall complexity is a combination of three elements: (i) the number of components, (ii) the patterns of the connections (which components are related), and (iii) the nature of the connections (the complexity of the calculations determining the relationships). These elements are also called size, connectedness, and calculation complexity, respectively. James et al. (1997) in the context of combined sewer overflow modeling, divide complexity into two groups: (i) complexity due to process disaggregation and (ii) complexity due to spatial discretization. They argue that, as a general rule, the simplest model that achieves the goals of the study should be used. Component processes require specific input parameters, the more detailed the more parameters required. Complexity arises when there is a large number of processes and interactions, when the relationships are non-linear, and where there are non-holonomic constraints (e.g. if a pump station in a drainage system switches on or off without regard to the system as a whole). The present writers propose simple definition, that model complexity be seen as a measure of both the number of processes activated and the number of subspaces (pipes and facilities) coded in the data file. In other words, that complexity be defined as the product of (i) the number of spatial sub-spaces modeled, (ii) the mean number of processes modeled in each, and (iii) the mean number of input parameters required for each process - in other words a measure of the total number of uncertain environmental parameters in the input data file (James, 1999). Complexity is thus related to the size of the input data file, excluding the meteorological driving input time series. where

We may write model complexity $\mathrm{C}=\mathrm{N}_{\mathrm{s}} \cdot \mathrm{N}_{\mathrm{pr}} \cdot \mathrm{N}_{\mathrm{pa}}$

$\mathrm{N}_{\mathrm{s}}$ = number of spatial sub-spaces modeled,

$\mathrm{N}_{\mathrm{pr}}=$ mean number of processes modeled in each,

$\mathrm{N}_{\mathrm{pa}}$ = mean number of input parameters required for each process. This total number of parameters does not include those relating to or the input time series itself (e.g. rainfall or inflows) that drives the system model.

Disaggregation is the intellectual procedure of dividing a real system into its theoretical component processes and sub-spaces. Aggregation is the reverse, the procedure of averaging a larger number of processes and sub- 
spaces into a smaller number of lumped sub-spaces and processes - the model is then said to be less complex. Consider the following example, a lake which has rain as input and stream flow as output, and whose water level changes from time to time.

1. One could consider all of the Great Lakes acting as one tank. Call this model GL1; here dx, dy are about $1000 \mathrm{~km}\left(10^{6} \mathrm{~m}\right)$ or more.

2. Or we could consider the Great Lakes as a networked system of six lakes, linked sequentially: Lakes Superior and Michigan both flow into Lake Huron, which then flows into Lake St Clair, which then flows into Lake Erie, which then flows into Lake Ontario, which finally outflows at Kingston. Call this model GL6; now dx, dy are now about $100 \mathrm{~km}\left(10^{5} \mathrm{~m}\right)$. We could say that GL6 is six times more complex than GL1.

3. Now consider modeling in more detail in each of all the areas, for instance several thousands of the pipes in your city (in our case, Guelph) alone. Call this GL1000; dx will now be about $0.1 \mathrm{~km}$ $\left(10^{2} \mathrm{~m}\right)$. Such a model could be said to be $10^{3}$ times more complex than GL1.

The higher the spatial resolution, the faster the frequency of the processes involved, and the finer the time resolution dt should be. In the real Great Lakes system, the response time of outflow at Kingston is long, and outflow varies only slightly on a monthly basis. The system responds to cycles of years, e.g. of wetter than average weather. Individual thunderstorms have no measurable impact. We could set $d t=1$ month (ca $2.5 \times 10^{6} \mathrm{~s}$ ). Small gutters and pipes in Guelph respond strongly to short thunderstorms, even if they last only 10 minutes. Here dt is about 1 minute $\left(6 \times 10^{1} \mathrm{~s}\right)$. Flow within the pipe is an even higher frequency process than that modeled in $\mathrm{G} 1000$, and now $\mathrm{dt}=10 \mathrm{~s}$ or less (perhaps as little as $1 \times 10^{\circ} \mathrm{s}$ ). If transients are involved in a surcharging pipe, then $\mathrm{dt}=0.1 \mathrm{~s}$ or less (perhaps as little as $1 \times 10^{-2} \mathrm{~s}$ ). So we see that choosing a coarse spatial resolution controls the time step, through the speed of the processes involved. In turn, choosing the time step controls the processes that will be involved. E.g. little ripples on the surface of the water have a natural frequency of about $10 \mathrm{~Hz}$. Their amplitudes would be averaged to zero if $\mathrm{dt}=$ 1 minute or more. Similarly, a short sharp thunderstorm would have a negligible rain intensity if averaged over a year.

Assessment of the decision support required for reducing uncertainty due to poor user performance is the subject of our current work. PCSWMM was set up to support complex modeling, e.g. $10^{5}$ parameters for Washington DC. Complex models cost more to set up than do simple models, and this relationship is now coded into PCSWMM2002. The effort and analytical cost for every one of the several thousand different parameters required for input has been 
determined and coded into the program. Uncertainty in parameter estimates has also been estimated. Part of this work requires a standard sensitivity analysis and a standard optimisation or calibration procedure, which have also been implemented in PCSWMM2002, together with a linear error analysis.

In this wide-ranging discussion, an obvious question which arises is: what level of complexity is best suited for the need at hand? In the present case we are considering learning and teaching, and it seems evident that users require models of systematically increasing complexity as their knowledge of the underlying processes develops and improves. In other words an introductory model for novices may be coarse and simple, while an advanced model for experts may be fine and complex - thus optimal complexity changes with experience. Can one build a model such that its complexity keeps pace with the advancing knowledge of the developing expert, and what kind of construction will most accelerate that learning process?

If experience, which shows that achievable modeling accuracy depends more on user quality than it does on model structure, is correct, then a GDSS that facilitates improved user performance is essential. Such a GDSS should include tools for context-sensitive help, sensitivity and error analysis, and an aggregation wizard for optimizing model complexity. For a review of GDSS, see Ostrowski and James (1998).

The selected GDSS, PCSWMMGIS2000 (James and James, 1995 \& 1999; James et al., 2000), provides a large array of file management; model sensitivity, error and calibration; output interpretation/presentation; and reference tools for urban drainage modeling. Some additional tools directly relevant to the thrust of this chapter are: model process aggregation; on-line animated tutorials; and a contextual help system exceeding 60,000 words.

In the next section we discuss our experience with the instructional ILE described above, and comment on user performance improvement.

\subsection{Discussion}

\section{Heuristics:}

Guidelines or heuristics for designing the courses (e.g. should each module comprise the work of one teaching week, or one class?) were derived from evolving experience, and the sound decision-making inherent in collaborating with four other Professors. One module per fortnight per course seems to be a good module size and frequency. This impression was confirned by the course evaluations - for this dual graduate course the evaluations were the best received by the authors so far in about 35 years experience (many combinations were tried over the years). 
Varied participating professors:

Students seemed to generally respond well to having modules offered by different instructors. Experiencing first-hand the knowledge of established experts is evidently hard to beat. In a course of six modules, probably two, three or even four professors would be better than one. The problem of course lies in getting five professors to complete their share in good time. However, an important point is that the professors should enthuse about this innovative teaching task, be great with students, and have attractive Internet persona.

\section{Selection of models:}

During the course students work on mathematical models which they download. Of course models used must be familiar to the instructors, and freely available to participating students. However not all commercial packages offer demonstration releases suitable for systematic work. Moreover, some packages need for their proper functionality other software (such as GIS, AutoCAD, databases). PCSWMMGIS2000 is made available to students and with it, no further GIS software is required .

\section{Student selection:}

Participating students were not selected by any formal or centrally coordinated process. Students enroll in the appropriate graduate course at their own University under the advice of the participating professor (Bob Pitt at the $U$ of Alabama, Manfred Ostrowski at Darmstadt Technological University, Neil Armitage at the University of Cape Town, and Isobel Heathcote or Bill James at the University of Guelph). We did not as a group select students, nor did we have any pre-selection criteria. Several students did not complete either course, so we believe that the course set its own rather high standards. At Guelph the professors involved tend to allow any enrolled graduate student to participate, but not to audit the course, with the warning that the courses are difficult. There should be no problem for any graduate students around the world to participate. If the graduate students' University is part of the Europe-wide Erasmus arrangement, then that student evidently can register through the Technical University of Darmstadt for no additional fee, and without any prior approval. If not, the student can apply to the University of Guelph to take the course remotely. This likely will cost money and may involve some bureaucracy. A better arrangement is for the graduate students' advisor to email to the authors a request to collaborate. We would then arrange the new shared instructional load. Note that, apart from the live lectures on the home campus, most of the real class-oriented personal instruction takes place through the list server. 
Peer evaluation:

In this dual course, self and peer evaluation worked better than we expected. Note that evaluation is not the same thing as grading. Students did not grade any work, grading was done by instructors. Admittedly our expectations were not high, and in their self-evaluation most students tended to downgrade their own work. We found that their evaluations were useful and confirmed most instructors' evaluations. On the other hand the authors have consistently found class-wide peer-grading to be unreliable. Students in our experience seem to value relationships highly, too high to be objective, and one cannot blame them. Furthermore their standards are at least as variable as those of their teachers. In our opinion, peer grading is not worth the effort; instead we asked students to read their peers' work carefully, and just comment on what they liked about it, or comment on whether they noticed anything noteworthy, missing or strange - just to prove that they had read the work critically. We did not ask students to assign a symbol to the work of their peers. In this way peer review can be handled with ease and in a small class of graduate students, can significantly enhance learning.

\section{User performance improvement:}

Part of this work that we could not readily substantiate relates to how user performance improvement was measured. We are uncertain whether this question can be answered objectively. We found that the web courses better integrate lessons, learning and design, and hence could lead to significant improvement of user performance. Undoubtedly students showed improvement in web group model participation skills, in their readiness to analyze and solve problems in more effective ways, and in their commentaries. On the other hand, students value personal contact in the lecture format, which condition we met by having weekly lectures for our own students on campus.

\section{Use of student feedback:}

Comments and/or the results of the work of students were taken into account in the development and improvement of PCSWMMGIS. We work as follows: we deal mostly in email and user support is in that medium. Emails and responses are archived (we currently have 794 items in one folder for the two graduate courses) then carefully catalogued and indexed and later form part of an extensive Q\&A subsystem. We do this meticulously to ensure that future questions are not repeated and answered again ab initio. Using a good programmer, the required improvements can be immediately implemented as improvements to the model help sub-system. PCSWMMGIS2000 was written from this philosophy - to expose uncertainty (in contrast to some other commercial packages, which avoid exposing the uncertainties). 
Financial support:

No central finances were involved. Other than the fact that the five professors all get paid for lecturing, no other funding was involved. Old fashioned as it sounds, the methodology was developed by us all purely for the love of learning. The author paid personally for all the required computer support and software. The other professors also contributed their copyrighted material without cost, each professor retaining copyright of course. Students paid their own graduate course registration fees at their home Universities, usually not very much.

\subsection{Conclusions}

Asynchronous (ILE's are now ubiquitous. We designed an ILE that removed the distinctions between instruction, learning and design (work). We also focused on model user performance improvement. Innovative methodology included:

1. use of models varying in support for user performance improvement,

2. lively use of a class list server,

3. presentation of copious course materials on the web,

4. submission and grading of assignments openly on the web,

5. self-evaluation,

6. FTP sites for downloading models and documentation,

7. global problems and applications of models, and

8. exploitation of the web and its resources.

It is still too early to draw hard conclusions, but we found that our ILE comprised an interesting test bed for the evolution of web-based design technologies. An important concern here is that model user performance needs to be improved and evaluated objectively.

Did the web graduate courses provide useful information for the evolution of models for improving user performance in the design of benign urban water systems? We think that the answer is affirmative, but we have not yet conducted an experiment to answer the question objectively. We are working on it.

\section{Acknowledgments}

We thank our colleagues Bob Pitt, Isobel Heathcote, Manfred Ostrowski and Neil Armitage for participating in this innovative graduate instruction; all participating graduate students for helping us to understand the pedagogic 
stumbling blocks inherent in a synchronous ILE; and all our colleagues mostly in Europe who contributed to the email discussion of an earlier draft of this chapter. Provision of web pages on the school server was made possible by the School of Engineering at the University of Guelph. The discussion was distilled from a number of emailed contributions of the first version of this chapter that was initially published on the web. We are grateful to the contributors for the nine questions posed.

\section{References}

Ambrose, R.B. et al. 1991. WASP4 - A hydrodynamic \& water quality model - model theory, user's manual, and programmer's guide. Georgia, EPA.

Beven, K. 1993. Prophecy, reality and uncertainty in distributed hydrological modeling. Adv. in Water Resources, vol.16, p.41-51

Brooks, R.J, and Tobias, A.M. 1996. Choosing the best model: level of detail, complexity, \& model performance. Mathematical \& Computer Modeling, vol.24, n.4,p.1-14

Flood, R.L., and Carson, E.R. 1993. Dealing with complexity: an introduction to the theory \& application of systems Sci. New York, Plenum, 280p.

James, W., 1999. Optimal complexity framework for models of benign urban water systems - asynchronous learning/design \& user performance using a web group decision support shell. CSCE 14th Can. Hydrotech. Conf., Regina, Sas. pp 599606.

James, W., T. El-Hosseiny and H.R. Whiteley. 1997. "On the Optimization of Uncertainty, Complexity and Cost for Modeling Combined Sewer Systems." Journal of Water Management Modeling R200-08. doi: 10.14796/JWMM.R200-08.

James, W; James, W.R.C.; Huber, W.C.; and Dickinson, R.E.; 2000. Water Systems Models: Hydrology -user's guide to SWMM4 runoff and supporting modules. Guelph, CHI, 6th printing.

James, W., and James, W. R. C. 1999. Integration of design models with GIS, the web $\&$ group decision support. CSCE 14th Can. Hydrotech. Conf., Regina, Sas.. pp 589-598.

James, R. and W. James. 1995b. "An Error-Control Decision Support System for SWMM." Journal of Water Management Modeling R183-21. doi: 10.14796/JWMM.R183-21.

James, W. and Ostrowski, M.W. (1998) Lessons learned during a first international graduate course on sustainable urban systems modeling using asynchronouslearning on the web. UDM '98, London, September 1998

Nihoul, J.C.J. 1998. Optimum complexity in eco-hydrodynamic modeling: an ecosystem dynamics standpoint. J. of Marine Systems, vol.16, p.3-5.

Ostrowski, M. W. and James, W. 1998. Requirements for group decision support systems (GDSS) for more sustainable urban water management. UDM '98, 4th Int. Conf. On Developments in Urban Drainage Modeling. London pp. 569-578. 


\section{APPENDIX A: Assignment A3 (model complexity)}

Module M3 merges (a) modeling, (b) GIS, and (c) urban drainage infrastructure. You are required to post a web page that summarizes your consideration of model complexity in terms of spatial and temporal discretization, and process disaggregation, and of how these concerns are closely tied to the development of input data files, since spatio-graphical data is the essence of urban water systems modeling. Your web page should prove that you understand the terminology and concepts of GIS in urban storm water systems modeling. A huge amount of reference material is available on this subject on the web and in technical libraries in books, monographs, conference proceedings, and journals, but you do not need to spend much time searching there because reading this module will cover most of your needs. The following suggestions are offered:

1. Discuss model complexity and postulate different needs for models of varying complexity. What levels of complexity are relevant to typical design problems in your area? Show that varying the model complexity requires conceptualization of the real problem at various degrees of spatial resolution. This implies activation of different physical processes, and requires input data files of varying size. Show that GIS could help in building data files because of its relationship to external databases, and its inbuiltutilities.

2. Briefly describe urban storm water systems in your area and how they function. To help you, browse through the design tasks involved in the design of urban water infrastructure used in my undergraduate class by clicking here, and through my rough lists of urban water infrastructure, by clicking here. Please read through all of the lists and make sure that you know what each device is. If you are uncertain please check my vocabulary or get the relevant book from your library and read about the devices in question. After you are certain that you know what all the devices are, create your own local list. If possible by all means insert as many images of local infrastructure as you can (or borrow from my aesthetic infrastructure page).

3. You could try running some simple data files of varying complexity for your favorite model, and summarizing the results - this task only for the way keen nerds. 